Revised version

LU TP 97-17

September 14, 2018

\title{
Local Interactions and Protein Folding: A Model Study on the Square and Triangular Lattices
}

\author{
Anders Irbäck円 and Erik Sandelin円 \\ Complex Systems Group, Department of Theoretical Physics \\ University of Lund, Sölvegatan 14A, S-223 62 Lund, Sweden \\ http://thep.lu.se/tf2/complex/
}

Submitted to Journal of Chemical Physics

\begin{abstract}
:
We study a simple heteropolymer model containing sequence-independent local interactions on both square and triangular lattices. Sticking to a two-letter code, we investigate the model for varying strength $\kappa$ of the local interactions; $\kappa=0$ corresponds to the well-known HP model [K.F. Lau and K.A. Dill, Macromolecules 22, 3986 (1989)]. By exhaustive enumerations for short chains, we obtain all structures which act as a unique and pronounced energy minimum for at least one sequence. We find that the number of such designable structures depends strongly on $\kappa$. Also, we find that the number of designable structures can differ widely for the two lattices at a given $\kappa$. This is the case, for example, at $\kappa=0$, which implies that the HP model exhibits different behavior on the two lattices. Our findings clearly show that sequence-independent local properties of the chains can play an important role in the formation of unique minimum energy structures.
\end{abstract}

\footnotetext{
${ }^{1}$ irback@thep.lu.se

2 erik@thep.lu.se
} 


\section{Introduction}

Natural proteins fold into unique compact structures in spite of the huge number of possible conformations [1]. It is widely believed that for most single domain proteins, the native structure is the global free-energy minimum [2, but the mechanism that determines the structure is still not understood. It is also not known whether the property of having a unique native structure is common or rare among random polypeptides. It is therefore tempting to find out under which conditions and to what extent unique native structures appear in simple heteropolymer models.

In recent years there has been an increasing interest in simple statistical-mechanical models for protein folding [3]. Most of the models that have been studied are lattice-based with contact interactions only. Such models focus on heterogeneity as the primary force which drives the formation of unique native structures, and it has been found that the degree of heterogeneity plays an important role. For example, for the cubic lattice, it has been shown that it is possible to design twenty-letter sequences that have unique native structures, whereas two-letter sequences with this property seem to be rare [4, 5]. However, a limitation of these models is that local interactions are neglected, and such interactions might be important not only for the local structure of the chains, as illustrated by a recent study of a simple three-dimensional off-lattice model [5]. This model, with a simple two-letter code, was studied both with and without sequence-independent local interactions. In the presence of these interactions, it was shown that it is possible to find sequences that have compact, well-defined native structures. Without the local interactions, no such sequences were found. In this paper we investigate the effects of local interactions in some detail in two-dimensional lattice models using a two-letter code.

Our starting point is the HP model of Lau and Dill [7], where the monomers are either hydrophobic $(\mathrm{H})$ or hydrophilic/polar $(\mathrm{P})$. This model contains no explicit local interactions, but the underlying lattice can be thought of in terms of local interactions. In order to study the influence of the lattice, we compare the behavior of the model on the square and triangular lattices. Our calculations are based on exhaustive enumerations of the full conformational space, for chains containing up to eighteen monomers on the square lattice and up to thirteen monomers on the triangular lattice. The triangular lattice has the advantage over the more widely used square lattice that it does not exhibit the well-known even-odd problem - on the square lattice it is impossible for two monomers at an even distance along the sequence to form a nearest-neighbor contact.

The properties of the HP model on the square lattice are known in some detail from previous studies [8]. In particular, it has been shown that about $2 \%$ of all HP sequences possess nondegenerate ground states on this lattice [9, 10]. It turns out that such sequences are much more rare on the triangular lattice. There is, for example, no 13-mer with non-degenerate ground state on this lattice.

Having seen this, we turn to a simple extension of the HP model which contains explicit sequenceindependent local interactions. We study this model for varying strength $\kappa$ of the local interactions, focusing on the set of all ground states that are non-degenerate and separated from the rest of the states by a sufficiently large energy gap. Sequences having such ground states can design the corresponding structures, and the number of sequences that can design a given structure will be called the designability of this structure [11]. Every structure that can be designed by at least one sequence will be called designable. 
We find that the number of designable structures is strongly $\kappa$-dependent, and that it can differ widely for the two lattices at a given $\kappa$. The difference is particularly large at $\kappa=0$, which corresponds to the HP model. However, the maximum numbers of designable structures for the two lattices are comparable, and for both lattices there is a pronounced peak in the number of designable structures at $\kappa=-0.5$. At this $\kappa$ the typical designable structure is compact with many turns. Focusing on maximally compact structures, we study the designability of individual structures at this $\kappa$. We find that the designability tends to be much higher on the square lattice. On this lattice we find that there are certain compact structures which can be designed by a very large number of sequences. This finding is in line with the results of $\mathrm{Li}$ et al. 11]. However, it is important to note that the results for the triangular lattice are different, which indicates that the emergence of such structures to some extent is related to the even-odd problem.

Our results clearly show that sequence-independent local interactions can play an important role in the formation of unique minimum energy structures. Although our study is confined to two dimensions, we expect this to hold in three dimensions as well. In fact, one may expect such interactions to be even more important in three dimensions, where the flexibility of the chains is greater.

\section{Methods}

The chains studied are linear and self-avoiding, and contain two monomer types, $\mathrm{H}$ and $\mathrm{P}$. A sequence is specified by a choice of monomer types at each position on the chain, $\left\{\sigma_{i}\right\}$, where $\sigma_{i}$ takes the values $\mathrm{H}$ and $\mathrm{P}$ and $i$ is a monomer index. A structure is specified by a set of coordinates for all the monomers, $\left\{\mathbf{x}_{i}\right\}$, and the bend angle formed by sites $\mathbf{x}_{i-1}, \mathbf{x}_{i}$ and $\mathbf{x}_{i+1}$ will be denoted by $\theta_{i}$. The energy of a structure is given by sequence-independent local interactions and sequence-dependent nearest-neighbor contact interactions,

$$
\begin{aligned}
E & =\kappa E_{L}+E_{G} \\
E_{L} & =2 \sum_{i=2}^{N-1}\left(1-\cos \theta_{i}\right) \\
E_{G} & =\sum_{1 \leq i<j \leq N} \epsilon_{\sigma_{i} \sigma_{j}} \Delta\left(\mathbf{x}_{i}-\mathbf{x}_{j}\right)
\end{aligned}
$$

where $\Delta\left(\mathbf{x}_{i}-\mathbf{x}_{j}\right)=1$ if $\mathbf{x}_{i}$ and $\mathbf{x}_{j}$ are nearest neighbours on the lattice but $i$ and $j$ are not adjacent positions along the sequence, and $\Delta\left(\mathbf{x}_{i}-\mathbf{x}_{j}\right)=0$ otherwise. The energy depends on three parameters $\epsilon_{\mathrm{HH}}, \epsilon_{\mathrm{HP}}$ and $\epsilon_{\mathrm{PP}}$ which will be held fixed throughout the paper. Following Lau and Dill [7], we take

$$
\epsilon_{\mathrm{HH}}=-1 \quad \epsilon_{\mathrm{HP}}=\epsilon_{\mathrm{PP}}=0
$$

The remaining parameter $\kappa$ determines the strength of the local interactions. For $\kappa=0$ the model is identical to the HP model, the energy being given by minus the number of topological HH contacts (two monomers $i$ and $j$ are in topological contact if $\Delta\left(\mathbf{x}_{i}-\mathbf{x}_{j}\right)=1$ ).

In our calculations we focus on the energy spectrum. For a given number of monomers, $N$, we compute this for all the $2^{N}$ possible sequences, by numerical enumeration of the full conformational space. In this way we determine all sequences having a ground state which is non-degenerate and 
separated from the rest of the spectrum by a sufficiently large energy gap. We say that such a sequence can design its ground state structure, and each structure that can be designed by at least one sequence will be called designable. The number of designable structures will be denoted by $D_{N}$ for chains with $N$ monomers.

These definitions involve a parameter $\Delta E$; the gap between the ground state and the next lowest level is required to be greater than or equal to $\Delta E$. The choice of this parameter is somewhat arbitrary. We tested several different values and decided to use $\Delta E=1$, corresponding to the energy of one HH contact. Small changes of $\Delta E$ leads to qualitatively similar results.

The gap criterion is important when studying general $\kappa$. To illustrate this, let us consider $N=13$ chains on the triangular lattice. Here one finds that all ground states are degenerate at $\kappa=0$, while 4328 of the sequences have non-degenerate ground states at small, positive $\kappa$. However, each ground state becomes effectively degenerate for sufficiently small $\kappa$, since all the gaps vanish in this limit.

Our choice of gap criterion implies that we look for ground states corresponding to a single structure on the lattice. For longer chains it would probably be more relevant to consider the gap between the ground state and the lowest of all states with little structural similarity to the ground state [12, 13, 14]. In principle, it would be more appropriate to formulate the gap criterion in terms of a normalized gap $\Delta E / E_{\kappa}, E_{\kappa}$ being a $\kappa$-dependent energy scale. However, the variation of $E_{\kappa}$ can, for our purposes, be neglected in the $\kappa$ region that is of primary interest (small and moderate $|\kappa|)$ ).

The total number of sequences that can design structures will be denoted by $S_{N}$ for chains with $N$ monomers. In general $S_{N}$ is greater than $D_{N}$, since different sequences may have the same ground state structure. The difference between $S_{N}$ and $D_{N}$ is particularly large in the trivial limit $\kappa \rightarrow \infty$. In this limit, there is one structure, the rod-like structure which minimizes $E_{L}$, which can be designed by all sequences, so $S_{N}$ is equal to the size of the full sequence space while $D_{N}=1$. In the limit $\kappa \rightarrow-\infty$ the situation is similar but slightly different. On the triangular lattice there is again one structure, a zig-zag pattern, which can be designed by all sequences. This structure is the unique maximum of $E_{L}$. On the square lattice there are, by contrast, many different structures that maximize $E_{L}$.

\section{Results}

\subsection{The number of designable structures}

\subsection{1 $\kappa=0$}

We first consider the model in absence of the local interactions $(\kappa=0)$. Energy gaps can then take integer values only, so, with our choice of $\Delta E$, the gap criterion is met by all sequences with non-degenerate ground states. Hence, $S_{N}$ is here simply the number of $N$-mers with non-degenerate ground states. In Table 1 we show the quantities $S_{N}$ and $D_{N}$ for different $N$ on the square and triangular lattices. Our results for $S_{N}$ on the square lattice can be compared with those of Chan and Dill [9, 10] and are consistent with these. Also shown in Table 1 are the total numbers of different conformations, unrelated by simple symmetries, for different $N$. 


\begin{tabular}{|c|c|c|c|c|c|c|}
\cline { 2 - 7 } \multicolumn{1}{c|}{} & \multicolumn{3}{c|}{ square } & \multicolumn{3}{c|}{ triangular } \\
\hline & $\begin{array}{c}\text { No. of } \\
\text { conformations }\end{array}$ & $S_{N}$ & $D_{N}$ & $\begin{array}{c}\text { No. of } \\
\text { conformations }\end{array}$ & $S_{N}$ & $D_{N}$ \\
\hline 3 & 2 & 0 & 0 & 3 & 2 & 1 \\
4 & 5 & 4 & 1 & 12 & 2 & 2 \\
5 & 13 & 0 & 0 & 52 & 1 & 1 \\
6 & 36 & 7 & 3 & 228 & 0 & 0 \\
7 & 98 & 10 & 2 & 996 & 0 & 0 \\
8 & 272 & 7 & 5 & 4324 & 0 & 0 \\
9 & 740 & 6 & 4 & 18678 & 0 & 0 \\
10 & 2034 & 6 & 4 & 80345 & 2 & 2 \\
11 & 5513 & 62 & 14 & 344431 & 6 & 6 \\
12 & 15037 & 87 & 25 & 1472412 & 2 & 2 \\
13 & 40617 & 173 & 52 & 6279601 & 0 & 0 \\
14 & 110188 & 386 & 130 & & & \\
15 & 296806 & 857 & 218 & & & \\
16 & 802075 & 1539 & 456 & & & \\
17 & 2155667 & 3404 & 787 & & & \\
18 & 5808335 & 6349 & 1475 & & & \\
\hline
\end{tabular}

Table 1: $S_{N}$ and $D_{N}$ for different $N$ for the HP model $(\kappa=0)$ on the square and triangular lattices. Also shown are the total numbers of conformations for different $N$.

From Table 1 it can be seen that $S_{N}$ increases roughly linearly with the total number of sequences on the square lattice; the fraction of sequences having non-degenerate ground states varies between 2.1 and $2.6 \%$ for $12 \leq N \leq 18$. Over the same $N$ range the number of designable structures satisfies $0.23 S_{N}<D_{N}<0.34 S_{N}$, so on average each designable structure can be designed by $2.9-$ 4.3 sequences. These results contrast sharply with those for the triangular lattice, where $S_{N}$ and $D_{N}$ are much smaller. Also, there is no structure on the triangular lattice that can be designed by more than one sequence for $4 \leq N \leq 13$.

\subsection{2 $\kappa \neq 0$}

We now turn to general $\kappa$. In Fig. 1 1 we show the $\kappa$ dependence of $D_{N}$ for $N=13$. The large- $|\kappa|$ behavior of $D_{N}$ is trivial, as discussed above. However, as can be seen from Fig. 1, there is a small- $|\kappa|$ region where $D_{N}$ shows an interesting and strong $\kappa$ dependence. For both lattices there is a sharp peak at $\kappa=-0.5$. For the square lattice there is another, slightly higher peak at $\kappa=0$, corresponding to the HP model. Such a peak is missing for the triangular lattice, which leads to the big difference in the results for the HP model.

In order to study the character of the designable structures, we computed average values of the total number of topological contacts, $C$, which is a measure of compactness, and $E_{L}$ (see Eq. 2). In Fig. 2 these quantities are plotted against $\kappa$, using $N=13$. Each data point in this figure is an average over all the designable structures at a given $\kappa$. For $N=13$, the maximum value of $C$ is 6 on the square lattice and 14 on the triangular lattice, whereas the maximum value of $E_{L}$ is 22 

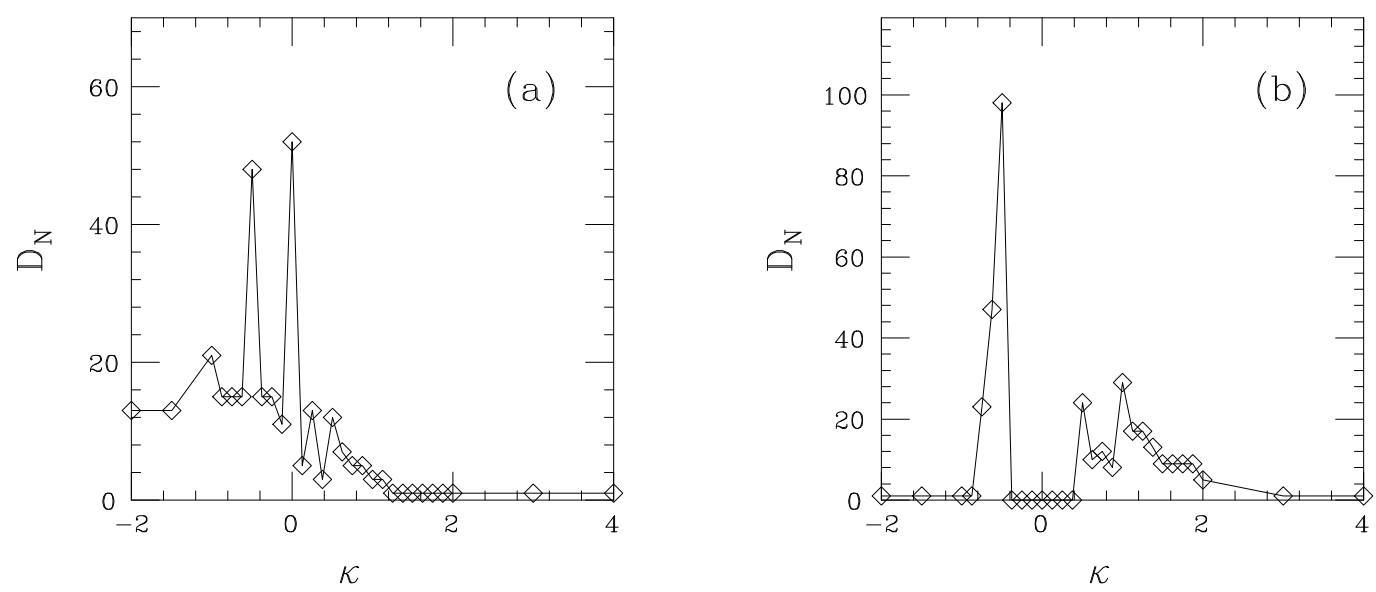

Figure 1: The number of designable structures, $D_{N}$, versus $\kappa$ for $N=13$, (a) square and (b) triangular lattice.

on the square lattice and 33 on the triangular lattice. From Fig. 2 it can be seen that $C$ and $E_{L}$ vary rapidly with $\kappa$. Both quantities are large at the peaks at $\kappa=-0.5$, showing that the typical designable structure at this $\kappa$ is compact with many turns.

A closer look at the designable structures at $\kappa=-0.5$ shows that roughly half of them are maximally compact, in the sense that they have maximum $C$; this holds for 19 out of 48 structures on the square lattice, and for 50 out of 98 structures on the triangular lattice. An example of a designable structure at $\kappa=-0.5$ which is not maximally compact is the zig-zag structure that maximizes $E_{L}$ on the triangular lattice. A structure such as this is less interesting than the maximally compact ones from the viewpoint of design. In our study below of the designability of individual structures, we focus on maximally compact structures.

Local properties of the designable structures, such as $E_{L}$, are strongly $\kappa$-dependent. To illustrate this, we show in Fig. 3 two designable structures on the triangular lattice corresponding to $\kappa=-0.5$ and $\kappa=0.5$, respectively. Both these conformations are maximally compact. Another important property which they share is that they display strong regularities in the local structure, reminiscent of the secondary structure in real proteins. The two conformations differ markedly, however, in the precise form of the local structure.

\subsection{The designability of individual structures}

So far we have classified the structures in a binary way as either designable or not. It is also interesting to see to what extent those structures that are designable differ in designability, the designability being defined as the number of sequences that can design a given structure.

Recently, Li et al. 11] studied the designability of individual structures in a HP-like model on the square and cubic lattices. These calculations were performed using the restricted conformational space consisting of all maximally compact structures, and the energy function was given by Eqs. 1-3 

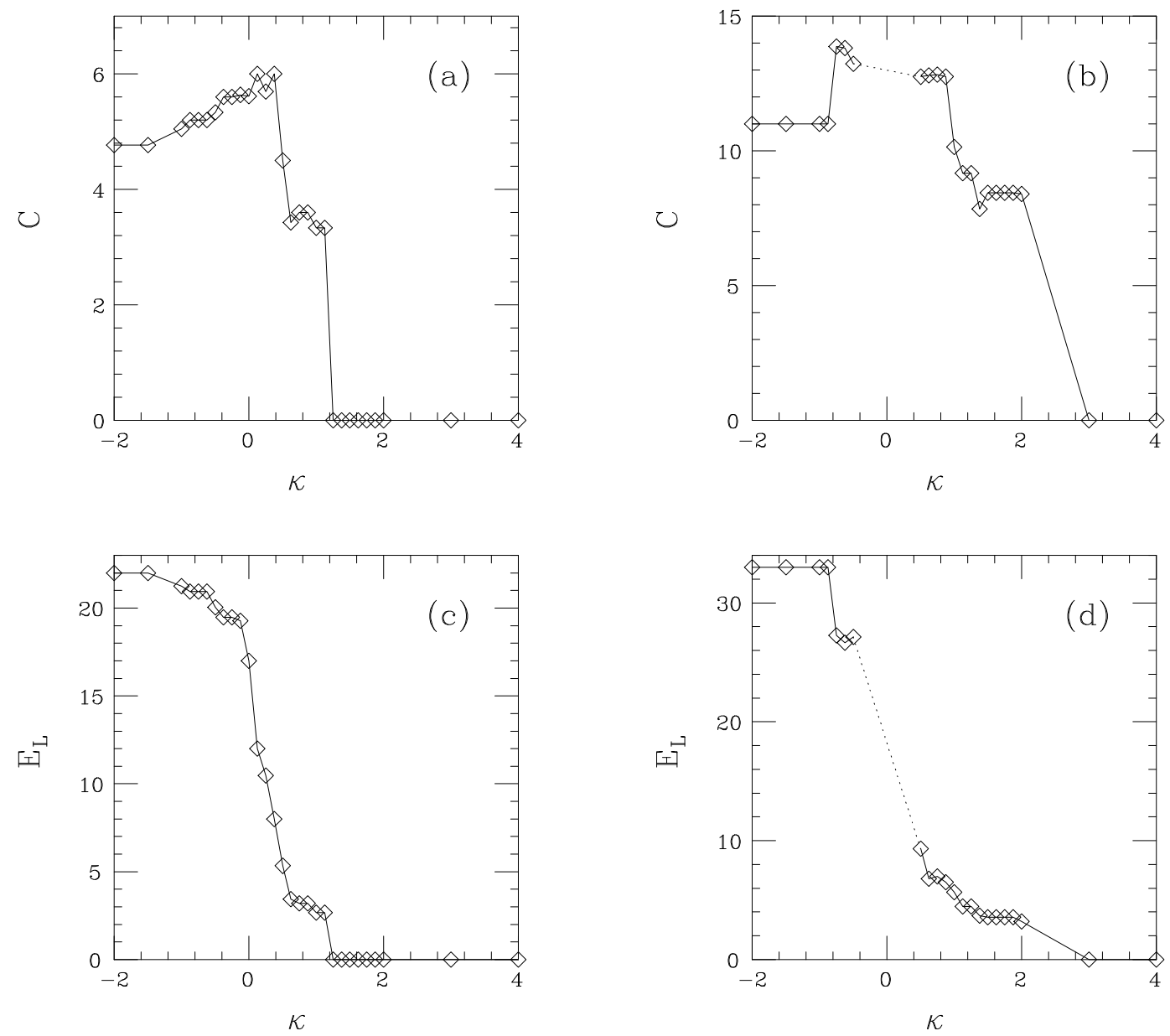

Figure 2: Averages of $C$ (the number of topological contacts) and $E_{L}$ (see Eq. 2) over the designable structures at different $\kappa$ for $N=13$ : (a) $C$, square lattice; (b) $C$, triangular lattice; (c) $E_{L}$, square lattice; (d) $E_{L}$, triangular lattice. For the triangular lattice, there are $\kappa$ values at which no designable structures were found (see Fig. 1). The lines connecting the data points are dotted in this region.

with $\epsilon_{\mathrm{HH}}=-2.3, \epsilon_{\mathrm{HP}}=-1$ and $\epsilon_{\mathrm{PP}}=\kappa=0$. Large variations in designability were observed. In particular, it was shown that certain structures can be designed by a huge number of sequences.

In order to test the generality of these findings, we have performed analogous calculations for our model using $\kappa=-0.5$ and $N=13$. The designability was computed for each of the maximally compact structures, as discussed above. Let us stress, however, that, in contrast to Li et al. [11], we base our definition of designability upon the full set of all possible structures rather than the subset of maximally compact structures.

Comparing our results for the square and triangular lattices, we find that the designability tends to be much higher on the square lattice. The average designability is 82.1 for this lattice, compared to 4.5 for the triangular lattice. The highest designabilities we measured are 590 and 12 , respectively, for the square and triangular lattices. The variations in designability are, as in the model studied 

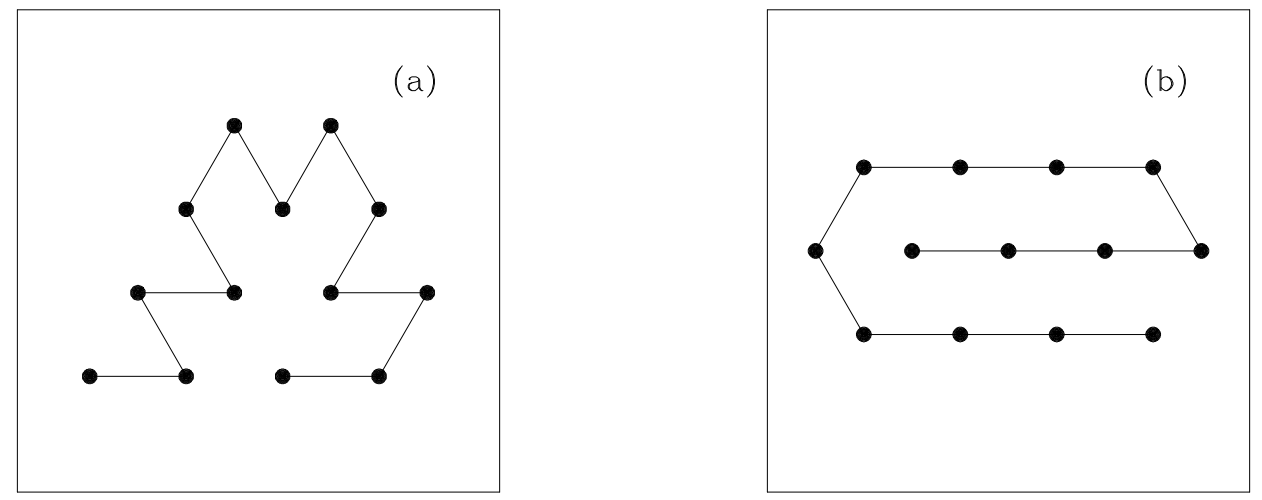

Figure 3: Examples of designable structures at (a) $\kappa=-0.5$ and (b) $\kappa=0.5$.

by Li et al. [11], very large on the square lattice.

The observed differences in designability for the two lattices are striking, but may be not surprising in view of the even-odd problem. The fact that certain contacts cannot be formed on the square lattice tends, for a given structure, to increase the degree of degeneracy with respect to the sequence degrees of freedom.

\section{Summary and discussion}

We have studied ground state properties of a simple heteropolymer model containing sequenceindependent local interactions. By enumeration of the full conformational space for short chains, we calculated the degeneracy of the ground state and the gap to the next lowest level for all possible sequences. In this way we obtained all structures that are designable. Our results show that the number of designable structures depends strongly on the strength $\kappa$ of the local interactions. Furthermore, we have seen that the behavior on the square and triangular lattices can be very different at a given $\kappa$. An example of this is the HP model $(\kappa=0)$. As our study of non-zero $\kappa$ shows, the difference in behavior of the HP model on the two lattices can to a certain degree be compensated for by introducing the local interactions. However, there seems to be at least one important difference between the properties on the two lattices. Our study of the designability of maximally compact structures shows that this tends to be much higher on the square lattice. Furthermore, on this lattice we find that, as in the model studied by Li et al. [11], there are certain compact structures that can be designed by a huge number of sequences. No such structures were found on the triangular lattice.

The effects of local interactions were recently studied in a simple three-dimensional off-lattice model with two-letter code [6]. Our findings are in good agreement with the results of this study, but may at sight seem to contradict the results obtained by Govindarajan and Goldstein [15]. It should therefore be stressed that the system studied by Govindarajan and Goldstein is very different from 
ours. They studied the behavior of optimized sequences in a model with a much higher degree of heterogeneity, in which the local interactions are sequence-dependent.

Starting with the work of Flory [16], there have been a number of studies of homopolymer models containing local interactions similar to those we have considered here. From the viewpoint of protein folding, the model studied by Kolinski et al. [17, 18 appears particularly interesting. This model contains, in addition to the local interactions, also attractive nearest-neighbor contact interactions. Its phase diagram for $\kappa>0$ exhibits coil and globule phases, as well as a folded low-temperature phase 19, 20]. The behavior of this model for $\kappa<0$ has to our knowledge not been investigated.

The HP model, without local interactions, has recently been utilized by Camacho and Schanke [21] in order to study the role of crosslinks in polymers. There have been several recent studies of this important issue based on Gaussian models [22, 23, 24. Camacho and Schanke took a different approach and examined the zero temperature limit of the HP model, where the surviving structures are those that have the maximum number of HH links. Their study was carried out using the square lattice, and it would be interesting to see to what extent the results remain unchanged on the triangular lattice. The behavior of the HP model on the triangular lattice has been studied previously in other contexts, recently by Seno et al. [25].

\section{Acknowledgements}

We would like to thank Carsten Peterson, Frank Potthast and Ola Sommelius for helpful comments on the manuscript, and Michele Vendruscolo for making unpublished data available to us. 


\section{References}

[1] T.E. Creighton, Proteins: Their Structure and Molecular Properties (Freeman, New York, 1993).

[2] C.B. Anfinsen, "Principles that Govern the Folding of Protein Chains", Science 181, 223 (1973).

[3] For a review, see M. Karplus and A. S̆ali, "Theoretical Studies of Protein Folding and Unfolding", Curr. Opin. Struct. Biol. 5, 58 (1995).

[4] E.I. Shakhnovich, "Proteins with Selected Sequences Fold into Unique Native Conformations", Phys. Rev. Lett. 72, 3907 (1993).

[5] K. Yue, K.M. Fiebig, P.D. Thomas, H.S. Chan, E.I. Shakhnovich and K.A. Dill, "A Test of Lattice Protein Folding Algorithms", Proc. Natl. Acad. Sci. USA 92, 325 (1995).

[6] A. Irbäck, C. Peterson, F. Potthast and O. Sommelius, "Local Interactions and Protein Folding: A 3d Off-Lattice Approach", J. Chem. Phys. 107, 273 (1997).

[7] K.F. Lau and K.A. Dill, "A Lattice Statistical Mechanics Model of the Conformational and Sequence Spaces of Proteins", Macromolecules 22, 3986 (1989).

[8] K.A. Dill, S. Bromberg, K. Yue, K.M. Fiebig, D.P. Yee, P.D. Thomas and H.S. Chan, "Principles of Protein Folding - A Perspective from Simple Exact Models", Protein Sci. 4, 561 (1995).

[9] H.S. Chan and K.A. Dill, "Sequence Space Soup' of Proteins and Copolymers", J. Chem. Phys. 95, 3775 (1991).

[10] H.S. Chan and K.A. Dill, "Transistion States and Folding Dynamics of Proteins and Heteropolymers", J. Chem. Phys. 100, 9238 (1994).

[11] H. Li, R. Helling, C. Tang and N. Wingreen, "Emergence of Preferred Structures in a Simple Model of Protein Folding", Science 273, 666 (1996).

[12] A. Sali, E. Shakhnovich and M. Karplus, "Kinetics of Protein Folding. A Lattice Model Study of the Requirements for Folding to the Native State", J. Mol. Biol. 235, 1614 (1994).

[13] J.D. Bryngelson, J.N. Onuchic, N.D. Socci and P.G. Wolynes, "Funnels, Pathways, and the Energy Landscape of Protein Folding: A Synthesis", Proteins: Struct. Funct. Genet. 21, 167 (1995).

[14] A.M. Gutin, V.I. Abkevich and E.I. Shakhnovich, "Evolution-Like Selection of Fast-Folding Model Proteins", Proc. Natl. Acad. Sci. USA 92, 1282 (1995).

[15] S. Govindarajan and R.A. Goldstein, "Optimal Local Propensities for Model Proteins", Proteins: Struct. Funct. Genet. 22, 413 (1995).

[16] P.J. Flory, "Statistical Thermodynamics of Semi-Flexible Chain Molecules", Proc. Roy. Soc. A 234, 60 (1956).

[17] A. Kolinski, J. Skolnick and R. Yaris, "The Collapse Transition of Semiflexible Polymers. A Monte Carlo Simulation of a Model System", J. Chem. Phys. 85, 3585 (1986).

[18] A. Kolinski, J. Skolnick and R. Yaris, "Monte Carlo Simulations on an Equilibrium Globular Protein Folding Model", Proc. Natl. Acad. Sci. USA 83, 7267 (1986). 
[19] S. Doniach, T. Garel and H. Orland, "Phase Diagram of a Semiflexible Polymer Chain in a $\theta$ Solvent: Application to Protein Folding", J. Chem. Phys. 105, 1601 (1996).

[20] U. Bastolla and P. Grassberger, "Phase Transitions of Semi-Stiff Polymer Chains", condmat/9705178 (1997).

[21] C.J. Camacho and T. Schanke, "From Collapse to Freezing in Random Heteropolymers", Europhys. Lett. 37, 603 (1997).

[22] M.P. Solf and T.A. Vilgis, "Statistical Mechanics of Macromolecular Networks without Replicas", J. Phys. A 28, 6655 (1995).

[23] J.D. Bryngelson and D. Thirumalai, "Internal Constraints Induce Localization in an Isolated Polymer Molecule", Phys. Rev. Lett. 76, 542 (1996).

[24] Y. Kantor and M. Kardar, "Conformations of Randomly Linked Polymers", Phys. Rev. E 54, 5263 (1996).

[25] F. Seno, M. Vendruscolo, A. Martian and J.R. Banavar, "Optimal Protein Design Procedure", Phys. Rev. Lett. 77, 1901 (1996). 